\title{
Análisis de Retorno Social de la Inversión en dos sistemas de apoyo a personas con gran discapacidad: asistencia personal y servicio residencial. Un estudio de caso ${ }^{1}$
}

\author{
Analysis of Social Return on Investment in two systems of support \\ for people with severe disabilities: personal assistance and residential \\ service. A case study
}

\section{Resumen}

En la actualidad existen diferentes alternativas orientadas a proporcionar apoyos a las personas con

discapacidad en su vida diaria, que requieren distintos despliegues y esquemas de aplicación de recursos. Estas alternativas, además, suponen diferentes impactos tanto en la vida de la misma persona con discapacidad como en su entorno próximo y en la sociedad. En este artículo se realiza un estudio de caso de una persona usuaria de asistencia personal en el Programa de Apoyo a la Vida Independiente (PAVI) a través de la Oficina de Vida Independiente (OVI) de la Comunidad de Madrid, en comparación con otro caso análogo en el ámbito residencial. El método de análisis utilizado se basa en el enfoque de Retorno Social de la Inversión (SROI, por sus siglas en inglés). Se especifican, asimismo, las decisiones adoptadas para la selección de los casos, los interlocutores consultados para recabar los conceptos y valores, las técnicas de recogida de información, las variables y fórmulas para el cálculo y la monetización. A pesar de su alcance limitado, es posible extraer conclusiones acerca del retorno social de la inversión en un "perfil tipo" que recibe servicios de asistencia personal frente a otro que recibe servicios en un entorno residencial.

Palabras clave

Asistencia Personal, coste-beneficio, política pública, discapacidad, diversidad funcional.

\begin{abstract}
At present, there are several alternatives to support daily life of people with disabilities, which require different resources: human, institutional, technical, material, financial, etc. In addition, these alternatives involve different impacts on both the life of people with disabilites and their immediate environment. This paper presents a case study that compares an user of personal assistance services of the Program for Independent Living (PAVI) with an user of a residential service. The study method used is based on the approach of Social Return on Investment (SROI). It also specifies the selection of cases, the partners consulted to gather concepts and values, data collection, variables and formulas for calculating and monetization. Despite its limited scope, it is possible to draw conclusions about the social return on investment in a "standard profile" receiving personal assistance services compared with a "standard profile" in a residential service.
\end{abstract}

\section{Keywords}

Personal assistance, cost-benefit, public policy, disability, functional diversity.

I. Este artículo se ha redactado a partir de un estudio más amplio realizado por iniciativa de la Oficina de Vida Independiente de ASPAYM Madrid, con financiación de la Consejería de Asuntos Sociales de la Comunidad de Madrid, a través de su Dirección General de Servicios Sociales.

\author{
Agustín Huete García \\ <ahueteg@usal.es> \\ Profesor Asociado de Sociología, \\ Universidad de Salamanca. \\ Director de Intersocial
}

\section{Martha Yolanda Quezada García}

<marthaquezada@intersocial.es>

Licenciada en Economía y Diploma de Estudios Avanzados (DEA) en Sociología

Para citar:

Huete García, A. y Quezada

García, M. Y. (20I4): “Análisis de Retorno Social de la Inversión en dos sistemas de apoyo a personas con gran discapacidad: asistencia personal y servicio residencial. Un estudio de caso". Revista Española de Discapacidad, 2 (I): 5I-69.

<http://dx.doi.org/I0.5569/23405 I04.02.01.03>

Fecha de recepción: I 5-O2-20I4 Fecha de aceptación: I7-06-20I4

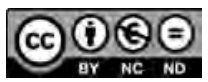




\section{Introducción}

El artículo 3 de la Convención Internacional sobre los Derechos de las Personas con Discapacidad (de ahora en adelante, CDPD) afirma los principios generales que han de servir como fundamento para el diseño de apoyos a las persona con discapacidad, entre ellos, " $e l$ respeto de la dignidad inherente, la autonomía individual, incluida la libertad de tomar las propias decisiones, y la independencia de las personas". Por su parte, el artículo i9 de la CDPD reconoce el derecho a vivir de forma independiente y a ser incluido en la comunidad y determina que se establezcan las medidas pertinentes que permitan que:

"Las personas con discapacidad tengan la oportunidad de elegir su lugar de residencia y dónde y con quién vivir, en igualdad de condiciones con las demás, y no se vean obligadas a vivir con arreglo a un sistema de vida específico;

Las personas con discapacidad tengan acceso a una variedad de servicios de asistencia domiciliaria, residencial y otros servicios de apoyo de la comunidad, incluida la asistencia personal que sea necesaria para facilitar su existencia y su inclusión en la comunidad y para evitar su aislamiento o separación de ésta;

Las instalaciones y los servicios comunitarios para la población en general estén a disposición, en igualdad de condiciones, de las personas con discapacidad y tengan en cuenta sus necesidades".

La Ley 39/2006, de I4 de diciembre, de Promoción de la Autonomía Personal y Atención a las personas en situación de dependencia establece una serie de servicios y prestaciones económicas que permitan mejorar la calidad de vida y la autonomía de las personas en situación de dependencia en España. El artículo I9 de dicha ley establece el derecho a una prestación económica de asistencia personal:
"La prestación económica de asistencia personal tiene como finalidad la promoción de la autonomía de las personas con gran dependencia. Su objetivo es contribuir a la contratación de una asistencia personal, durante un número de horas, que facilite al beneficiario el acceso a la educación y al trabajo, así como una vida más autónoma en el ejercicio de las actividades básicas de la vida diaria. Previo acuerdo del Consejo Territorial del Sistema para la Autonomía y Atención a la Dependencia, se establecerán las condiciones específicas de acceso a esta prestación.”

La Ley 39/2006 define la asistencia personal (art. 2.6.) como "un servicio prestado por un asistente personal que realiza o colabora en tareas de la vida cotidiana de una persona en situación de dependencia, de cara a fomentar su vida independiente, promoviendo y potenciando su autonomía personal". En palabras de un usuario receptor de servicios de asistencia personal:

"La asistencia me cubre todas las necesidades que tengo. Me tengo que levantar a las $6.30 \mathrm{~h}$ de la mañana, con lo cual, hay un asistente que duerme en mi casa, porque yo por la noche necesito cambios posturales, a lo mejor una o tres veces a la noche para evitar escaras y tal, me levanta, me lleva al trabajo,... Luego, hay otra persona que me recoge del trabajo, venimos para casa, hacemos la comida, comemos... Si hace falta salir, se sale, si hace falta comprar, se va, si hace falta estar en casa, se está... una vida normal".

Los años recientes han supuesto un giro en el paradigma de atención a las personas con discapacidad, planteando una transición desde esquemas de asistencia institucionalizada hacia servicios que promuevan la inclusión en la comunidad (Rodríguez Cabrero, 2009). Tal como expresa la Comisión Europea en la "Estrategia Europea sobre Discapacidad 20102020” (Comisión Europea, 2010):

"Estos derechos incluyen el derecho a la libre circulación, a elegir dónde y cómo se quiere vivir, y a tener pleno acceso a las actividades culturales, recreativas y deportivas." 
[La Comisión se dedicará a] "promover la transición de una asistencia institucional a una asistencia de carácter local ${ }^{2}$ mediante el uso de los Fondos Estructurales y del Fondo de Desarrollo Rural para respaldar la evolución de los servicios de asistencia locales y sensibilizar sobre la situación de personas con discapacidad alojadas en centros residenciales, especialmente los niños y las personas mayores;"

"La actuación de la UE apoyará actividades nacionales destinadas a: - lograr la transición de una asistencia institucional a una asistencia de carácter local, también mediante la utilización de los Fondos Estructurales y el Fondo de Desarrollo Rural, a efectos de la formación de recursos humanos y la adaptación de las infraestructuras sociales, el desarrollo de sistemas de financiación para ayudas personalizadas, la promoción de condiciones laborales adecuadas para los cuidadores profesionales y el apoyo a las familias y a los cuidadores no profesionales;"

A pesar del impulso que supone la CDPD como instrumento jurídico vinculante, y el cambio de los marcos doctrinales y normativos de atención en pro de servicios que favorezcan la inclusión comunitaria y la vida independiente, entre ellos la Asistencia Personal, basada en la demanda y enfoque del usuario ofrece una herramienta destacada, su implantación en nuestro país es todavía incipiente, con iniciativas muy relevantes, si bien con una escasa participación en términos cuantitativos respecto a otras modalidades de asistencia (Arnau et al., 2007). El servicio de asistencia personal, aunque reconocido por la Ley 39/2006, se encuentra poco desarrollado como dispositivo de apoyo dentro del Sistema para la Autonomía y Atención a la Dependencia (SAAD). Según datos del Instituto de Mayores y Servicios Sociales (IMSERSO, 2013), I.248 personas contaban en febrero de 2013 con la prestación económica de Asistencia Personal; esto supone sólo un o, I3 \% del total de prestaciones del SAAD. El 88,5\% de las personas que cuentan con esta prestación se encuentran en el País Vasco.

2. En inglés, "community-based care”.
Tabla 1. Personas con prestación económica de Asistencia Personal

\begin{tabular}{|l|c|}
\hline CCAA & $\begin{array}{c}\text { Total } \\
\text { personas }\end{array}$ \\
\hline Andalucía & 15 \\
\hline Asturias & 1 \\
\hline Castilla y León & 35 \\
\hline Castilla-La Mancha & 7 \\
\hline Cataluña & 17 \\
\hline Comunidad Valenciana & 2 \\
\hline Galicia & 41 \\
\hline Comunidad de Madrid & 25 \\
\hline Comunidad Foral de Navarra & 1 \\
\hline País Vasco & 1.104 \\
\hline Total & $\mathbf{1 . 2 4 8}$ \\
\hline
\end{tabular}

Fuente: IMSERSO (20I3).

\section{Metodología}

Existe una considerable tradición y variedad de propuestas metodológicas para el análisis del impacto de la inversión social y de los retornos que ésta produce. La estimación del impacto busca medir los resultados -en términos de cambios/efectos- que una determinada intervención social (plan/programa/proyecto) ha transferido a una población objetivo, en un determinado contexto. A grandes rasgos, el impacto puede medirse en términos de utilidad o eficacia (perspectiva cualitativa) o bien a partir de la del análisis de coste-beneficio de dicha intervención (perspectiva cuantitativa).

Independientemente de la orientación que se adquiera, el análisis de impacto resulta más completo si se añade el estudio del retorno de la inversión, en función de los resultados que en términos cuantitativos o cualitativos se pueden deducir de la intervención social que se estudia (Pena Trapero, 2009). 
En este trabajo se pretende medir el retorno económico de un usuario que recibe servicio de asistencia personal para personas con discapacidad, en comparación con otra forma de atención disponible en nuestra sociedad para la prestación de apoyos generalizados a personas con discapacidad física.

En concreto, se analizará el impacto económico de un usuario del Programa de Ayuda a la Vida Independiente (PAVI), de la Consejería de Asuntos Sociales de la Comunidad de Madrid, como modelo de servicio basado en asistencia personal, frente a un servicio de apoyo que también ofrece la Comunidad de Madrid, en concreto un servicio residencial. El modelo de estudio que se presenta es ampliable, a futuro, tanto en relación con la muestra de usuarios/as como hacia otros servicios y programas.

El impacto y su correspondiente retorno económico se calculan a partir de dos grandes dimensiones a considerar:

- La inversión pública que suponen los servicios.

- El retorno de esta inversión vía impuestos, seguridad social, creación de empleo, y otras fuentes de riqueza para el Estado.

\subsection{Retorno Social de la Inversión: el enfoque adoptado y sus fases}

El Retorno Social de la Inversión (SROI, o Social Return Of Investment, en inglés) es una metodología para la medición del impacto social de una inversión cualquiera. En su origen, el método SROI se creó a finales de los 90 por la Fundación $\mathrm{REDF}^{3}$ para calcular los beneficios

3. The Roberts Enterprise Development Fund es una entidad filantrópica radicada en San Francisco, orientada a crear oportunidades de empleo para personas que afrontan las mayores dificultades en este campo. Ante la constatación de que el valor económico y social creado por el sector no lucrativo no se monetizaba adecuadamente, calculaba y reconocía, la REDF diseñó una metodología orientada a registrar el "valor combinado", no sólo económico sino también de valores más amplios para la sociedad asociados a la inversión en diferentes proyectos, de manera objetivable y expresada en términos financieros. Más información en: <http://www.redf.org/>. para la sociedad de los programas sociales y de las empresas orientadas a la creación de empleo para personas con especiales dificultades de inserción laboral. En la actualidad es un método generalizado de cálculo del retorno sobre multitud de programas oficiales, sobre todo en el ámbito anglosajón (Estados Unidos, Reino Unido, Irlanda o Australia), y más allá de ellos, como referente metodológico aplicado a diferentes iniciativas y proyectos, a través de la Red Internacional del SROI ${ }^{4}$ (The SROI Network, 20I2).

Desarrollado a partir de un análisis tradicional de coste-beneficio y la contabilidad social, el análisis SROI evidencia cómo una organización (o programa, proyecto o iniciativa) crea valor, llegando a una expresión monetaria total del retorno que se crea por cada unidad invertida.

El coeficiente de SROI, por tanto, consiste en una comparación entre el valor generado por una iniciativa y la inversión necesaria para lograr ese impacto. Sin embargo, un análisis SROI no debe limitarse a un número, lo que en principio se utiliza para expresar el valor (The SROI Network, 20I 2). Por el contrario, presenta un marco para explorar el impacto social de una organización, en el que la monetización desempeña un papel importante, pero no exclusivo.

Los principios centrales del análisis SROI son los siguientes, Siguiendo a ECODES Alter Civites (2OI2) y The SROI Network (2OI2):

- Participación de los principales grupos de interés, o stakeholders.

- Creación de un mapa de impacto para ayudar a comprender los cambios e impactos que crean las organizaciones y/o iniciativas. El mapa de impacto pone de manifiesto la relación entre los recursos

4. La Red Internacional SROI es un organismo internacional cuyos objetivos son la promoción y el desarrollo del método SROI, velando por la mejora de esta metodología a través de la participación de sus socios. Hoy constituye la principal referencia sobre este método, como también lo es para el presente estudio. Más información en: <http://www.thesroinetwork.org/>. 
dedicados a determinadas actividades y los resultados de estas actividades.

- Reconocimiento de los límites de los impactos de un programa u organización asignando una parte de los resultados a otras organizaciones y /o circunstancias.

- Inclusión sólo de los impactos significativos en el análisis mediante una evaluación de materialidad.

- Asignación de valores económicos a todos los resultados para asegurar la inclusión de todos los temas relevantes para los grupos de interés, incluyendo los que carecen de un valor de mercado.

- Transparencia en la base metodológica del análisis, con claridad para la información y discusión con los grupos de interés.

Las fases en las que se aplica el análisis SROI, de manera resumida, son las siguientes:

- $\quad$ Fase 1: Establecimiento del alcance y la identificación de los grupos de interés. El primer paso es el establecimiento de límites claros sobre lo que se incluirá en el análisis SROI, quién va a participar en el proceso y cómo. Se determina el alcance y se identifican todos aquellos actores a los cuales puede impactar el proyecto.

- $\quad$ Fase 2: Recolección de información. Consiste en la aplicación de diversas herramientas y técnicas para recopilar información y datos que permitan medir el grado de cumplimiento de los resultados identificados durante la fase previa. Posteriormente se lleva a cabo un ejercicio de asignación de un valor monetario a los resultados cuyo valor es difícil de identificar, mediante estimaciones que permitan llevar a cabo el cálculo SROI. Es importante identificar indicadores de resultados e impactos y no sólo de procesos. Medir únicamente las salidas u outputs de actividades y/o procesos ofrece una visión parcial sobre el impacto total de la organización. Para ello se realiza una primera aproximación a la monetización de los indicadores y se recaba información sistemáticamente utilizando diferentes instrumentos.

- Fase 3. Resultados y determinación del impacto. Se evalúan y se elaboran estimaciones sobre el grado en el que los resultados identificados con anterioridad se deben a las actividades llevadas a cabo por el proyecto u organización objeto del análisis. El ejercicio sirve para ajustar los cálculos de los impactos teniendo en cuenta otros factores, personas u organizaciones que puedan influir en estos. Se calcula el SROI de acuerdo con la monetización planteada en la etapa previa; adicionalmente se culmina el mapa de resultados e impacto.

- Fase 4: Comunicación y uso. La última etapa consiste en la redacción de un informe y la comunicación de los resultados a los distintos grupos de interés de las organizaciones. Se preparan los informes y la estrategia de comunicación tanto interna como externa.

La metodología SROI se ha tomado como marco general de aplicación para el presente trabajo y se ha adaptado para realizar una aproximación comparativa a los dos casos típicos analizados de apoyo a personas con discapacidad, en las siguientes fases y tareas:

- Fase 1. Identificación de alcance y grupos de interés: Personas con discapacidad usuarias del servicio de asistencia personal y residencial, personas que prestan apoyo a las personas con discapacidad (familiares, voluntarios, asistentes personales...), gestores de este tipo de servicios.

- Fase 2. Recolección de información: Determinación de los perfiles tipo de los servicios de asistencia personal y residencial en la Comunidad de Madrid; matriz de registro de los ingresos, gastos, inversión e impacto; recogida de datos a través de 
entrevistas a las personas con discapacidad seleccionadas para el estudio.

- Fase 3. Resultados y determinación de impacto: Recursos invertidos por persona en estos servicios de apoyo, indicando resultados en términos de coste-beneficio, incluyendo el retorno social de la inversión. Cálculo del impacto.

- Fase 4. Comunicación y uso. Redacción de informes y otros documentos de difusión de información, como el presente artículo.

\subsection{Fuentes e instrumentos de recolección de información}

La recogida de información se ha realizado a partir de fuentes secundarias (documentales y estadísticas), así como mediante una serie de nueve entrevistas en profundidad a los perfiles tipo seleccionados, a personas que les prestan apoyos y a los gestores de cada uno de los servicios.

En total se han realizado tres entrevistas por cada perfil (persona con asistencia personal y persona con atención residencial): una persona con discapacidad, una persona que le presta apoyos y un gestor de servicios.

Se han realizado dos entrevistas más con perfiles de transición, es decir, personas con discapacidad que habían pasado de un servicio a otro con el objetivo de captar, en la medida de lo posible, algunos cambios en la estructura de ingreso-gasto a partir del cambio del servicio recibido.

Se ha realizado además una entrevista con un experto en tributos a fin de comprobar la adecuación de la matriz y decisiones analíticas, en especial, respecto a determinadas partidas relacionadas con la amortización de inversiones e impuestos.

En cada entrevista se han utilizado formularios estandarizados destinados a localizar la información precisa parar realizar la descripción y evaluación del impacto económico utilizando la metodología SROI.

Cabe destacar que aunque este estudio ha tenido un carácter económico, se ha procurado atender a aquellos aspectos que no se pueden monetizar fácilmente, pero que influyen en el bienestar de las personas con discapacidad y el entorno además de crear valor social.

El trabajo de campo se realizó entre el i 8 enero y el I 6 marzo de 20I3, según el esquema que presenta en la Tabla 2:

Tabla 2. Relación de entrevistas realizadas

\begin{tabular}{|c|l|c|}
\hline $\mathbf{N}^{\mathbf{2}}$ & Perfil & Fecha \\
\hline 1 & $\begin{array}{l}\text { Directora de un servicio } \\
\text { residencial }\end{array}$ & $\begin{array}{c}18 \mathrm{de} \\
\text { enero }\end{array}$ \\
\hline 2 & $\begin{array}{l}\text { Gestora del servicio de } \\
\text { asistencia personal }\end{array}$ & $\begin{array}{c}18 \mathrm{de} \\
\text { enero }\end{array}$ \\
\hline 3 & Usuario del servicio residencial & $\begin{array}{c}18 \mathrm{de} \\
\text { enero }\end{array}$ \\
\hline 4 & $\begin{array}{l}\text { Usuario de un Servicio de } \\
\text { asistencia personal }\end{array}$ & $\begin{array}{c}12 \text { de } \\
\text { febrero }\end{array}$ \\
\hline 5 & $\begin{array}{l}\text { Persona que ha tenido } \\
\text { asistencia personal y que ahora } \\
\text { está en una residencia }\end{array}$ & $\begin{array}{c}28 \mathrm{de} \\
\text { enero }\end{array}$ \\
\hline 6 & $\begin{array}{l}\text { Persona que ha vivido en una } \\
\text { residencia y ahora cuenta con } \\
\text { asistencia personal }\end{array}$ & $\begin{array}{c}29 \mathrm{de} \\
\text { enero }\end{array}$ \\
\hline 7 & Asistente personal & $\begin{array}{l}12 \text { de } \\
\text { febrero }\end{array}$ \\
\hline 9 & $\begin{array}{l}\text { Entrevista a un experto en } \\
\text { tributos }\end{array}$ & $\begin{array}{c}30 \text { de } \\
\text { enero }\end{array}$ \\
\hline
\end{tabular}

Fuente: Elaboración propia.

\subsection{Variables y fórmulas para monetización}

Se ha caracterizado el perfil tipo de los usuarios de cada uno de los servicios sobre los que se va a realizar el análisis de impacto, es decir, el Programa de Apoyo a la Vida Independiente (PAVI) y el servicio de centro residencial de la 
Fundación Lesionado Medular de la Comunidad de Madrid. Una vez identificado este perfil tipo, se ha seleccionado un caso aproximado al "tipo ideal o promedio" de cada uno de los servicios, a partir del cual se ha realizado el análisis de impacto.

Para determinar el análisis de impacto, se ha elaborado una matriz que registra los flujos económicos en términos de coste-beneficio, de forma que el impacto económico se calcula considerando:

- Inversión pública (gasto) por persona que supone el servicio (en este caso el servicio de asistencia personal y el servicio residencial).

- Inversión privada (de la propia persona): en medios humanos, materiales, etc.

- Retorno de esta inversión vía:

- Impuestos directos e indirectos.

- Cotizaciones a la Seguridad Social.

- Creación de riqueza: generación de consumo, empleo, dinamización de impuestos.

- Otros.

La Tabla 3 presenta la matriz de registro de estas variables y dimensiones.

Los criterios de aplicación en la matriz de registro y monetización son los siguientes:

1. Columna "Variables": incluye los diferentes conceptos sobre los que se realiza la estimación: el ingreso por empleo, los gastos relativos a actividades de la vida diaria así como los derivados por la discapacidad y los gastos que suponen los servicios de apoyo. Los datos que se incluyen en esta columna son reales, obtenidos en el trabajo de campo; no son estimaciones. Incluye:

- Ingresos que obtiene la persona con discapacidad: ya sea por empleo, prestaciones u otros rendimientos.

- Gastos que realiza la persona con discapacidad: relativos a actividades de la vida diaria así como específicos por discapacidad.
- Servicios de apoyo que recibe la persona con discapacidad: registra los gastos y retornos relacionados con el tipo de apoyo que recibe la persona (en este caso asistencia personal o residencia). Incluye:

- Construcción; inversión; infraestructura: Se considera la amortización a efectos de monetización, calculada con la siguiente fórmula: Amortización anual = coste de la obra / 33 (años promedio en que se considera amortizada la infraestructura según lo establecido en el Real Decreto I 777/2004, de 30 de julio, por el que se aprueba el Reglamento del Impuesto sobre Sociedades).

- Recursos humanos (atención directa): según información obtenida en el trabajo de campo.

- Recursos humanos (administración y gestión): según información obtenida en el trabajo de campo.

- Alquileres: según información obtenida en el trabajo de campo.

- Suministros, proveedores; ropa, equipamiento: según información obtenida en el trabajo de campo.

- Mantenimiento: según información obtenida en el trabajo de campo.

- Costes de formación: según información obtenida en el trabajo de campo.

2. Columna Base: La segunda recoge el importe para cada concepto de acuerdo con los datos recabados en e trabajo de campo, para cada uno de los ítems anteriores.

3. Columna Gasto: donde se registra el monto de la Inversión Pública (IP) en relación con los ingresos, la cobertura de gastos especiales por discapacidad así como destinada a los servicios de apoyo. Los datos que se incluyen en esta columna son reales, obtenidos en el trabajo de campo; no son estimaciones. Incluye: 
Tabla 3. Análisis del impacto. Matriz de registro y monetización

\begin{tabular}{|c|c|c|c|c|c|c|}
\hline \multirow{3}{*}{ Variables } & \multicolumn{6}{|c|}{ Impacto Erario público (Monetización) } \\
\hline & \multirow{2}{*}{$\begin{array}{l}\text { Gasto (Inversión } \\
\text { Pública o IP) }\end{array}$} & \multicolumn{5}{|c|}{ Retorno (R) } \\
\hline & & $\begin{array}{l}\text { Impuesto } \\
\text { directo }\end{array}$ & $\begin{array}{c}\text { Otros } \\
\text { impuestos }\end{array}$ & $\begin{array}{l}\text { Seguridad } \\
\text { Social }\end{array}$ & $\begin{array}{l}\text { Aportación de } \\
\text { riqueza }\end{array}$ & $\begin{array}{l}\text { Otros } \\
\text { retornos }\end{array}$ \\
\hline \multicolumn{7}{|l|}{ Ingresos para el usuario } \\
\hline Empleo & $\begin{array}{l}\text { Subvenciones, } \\
\text { exenciones } \\
\text { fiscales y en } \\
\text { Seguridad Social }\end{array}$ & IRPF & & $\begin{array}{l}\text { Empresa y } \\
\text { trabajador }\end{array}$ & & \\
\hline $\begin{array}{l}\text { Prestaciones Económicas: Pensión } \\
\text { contributiva de gran invalidez o } \\
\text { enfermedad, permanente absoluta, } \\
\text { permanente total; pensión no contributiva } \\
\text { de invalidez o enfermedad; prestación } \\
\text { familiar por hijo a cargo con discapacidad, } \\
\text { otras prestaciones }\end{array}$ & $\begin{array}{l}\text { Subvenciones, } \\
\text { exenciones } \\
\text { fiscales }\end{array}$ & $\begin{array}{l}\text { IRPF según } \\
\text { prestación }\end{array}$ & & $\begin{array}{l}\text { Según } \\
\text { prestación }\end{array}$ & & \\
\hline $\begin{array}{l}\text { Otros ingresos: inversiones, } \\
\text { rendimientos empresariales,... }\end{array}$ & $\begin{array}{l}\text { Exenciones } \\
\text { fiscales }\end{array}$ & IRPF y otros & & & & \\
\hline \multicolumn{7}{|l|}{ Gastos para el usuario } \\
\hline $\begin{array}{l}\text { Especiales por causa de discapacidad } \\
\text { (no servicios de apoyo): Tratamiento } \\
\text { médico/ atención sanitaria; adquisición de } \\
\text { medicamentos y otros productos sanitarios; } \\
\text { productos de apoyo/ ortopedia; adaptación } \\
\text { de vivienda; transporte (incluyendo aquí la } \\
\text { adaptación de su vehículo); otras }\end{array}$ & $\begin{array}{l}\text { Subvenciones, } \\
\text { exenciones } \\
\text { fiscales }\end{array}$ & IRPF y otros & IVA & & \multirow{2}{*}{$\begin{array}{l}\text { Consumo y } \\
\text { empleo indirecto }\end{array}$} & \\
\hline $\begin{array}{l}\text { Consumo: Alimentos y bebidas; vestir y } \\
\text { calzado; vivienda; agua; electricidad, gas, } \\
\text { combustibles; mobiliario, equipamiento, } \\
\text { gastos corrientes, conservación de la } \\
\text { vivienda; transportes; comunicaciones; } \\
\text { ocio, espectáculos; enseñanza; hoteles, } \\
\text { restauración; otros bienes y servicios }\end{array}$ & $\begin{array}{l}\text { Subvenciones, } \\
\text { exenciones } \\
\text { fiscales }\end{array}$ & & $\begin{array}{l}\text { IVA y } \\
\text { especiales }\end{array}$ & & & \\
\hline \multicolumn{7}{|l|}{ Servicios de apoyo en la vida diaria } \\
\hline $\begin{array}{l}\text { Construcción; inversión; infraestructura (Se } \\
\text { considera la amortización anual a efectos } \\
\text { de monetización) }\end{array}$ & Subvenciones & & IVA e IBI & & \multirow{8}{*}{$\begin{array}{l}\text { Consumo y } \\
\text { empleo indirecto }\end{array}$} & \\
\hline Recursos humanos (atención directa) & Subvenciones & IRPF & & $\begin{array}{l}\text { Empresa y } \\
\text { trabajador }\end{array}$ & & \\
\hline $\begin{array}{l}\text { Recursos humanos (administración y } \\
\text { gestión) }\end{array}$ & Subvenciones & IRPF & & $\begin{array}{l}\text { Empresa y } \\
\text { trabajador }\end{array}$ & & \\
\hline Alquileres & Subvenciones & IRPF & & & & \\
\hline $\begin{array}{l}\text { Suministros, proveedores: ropa, } \\
\text { equipamiento }\end{array}$ & Subvenciones & & $\begin{array}{c}\text { IVA y } \\
\text { especiales }\end{array}$ & & & \\
\hline Mantenimiento & Subvenciones & & $\begin{array}{c}\text { IVA y } \\
\text { especiales }\end{array}$ & & & \\
\hline Costes de formación & Subvenciones & & & & & \\
\hline \multicolumn{6}{|l|}{ Otros } & \\
\hline TOTAL SERVICIO & Total & Total & Total & Total & Total & Total \\
\hline
\end{tabular}

Fuente: Elaboración propia. 
- Subvenciones, exenciones y otros incentivos: que se traducen en gastos para el Estado, para financiar apoyos, ayudas técnicas, descuentos en impuestos, seguridad social, o cualquier otro apoyo por causa de discapacidad, que sea monetizable.

4. Columna Retorno: donde se calcula el retorno vía impuestos (IRPF, IVA), Seguridad Social, aportación de riqueza (a través del consumo y otros efectos indirectos). Las cifras de estas columnas son estimaciones realizadas a partir de la información obtenida en el trabajo de campo, o bien de las fuentes secundarias disponibles. Todas las estimaciones se calculan sobre las cifras reales obtenidas para cada perfil. Incluye el retorno hacia el Estado en forma de:

- IRPF: según tramos salariales establecidos por Hacienda, se estima un tipo medio del $2 \mathrm{I} \%$ para ingresos inferiores a 33.000 euros brutos anuales.

- IVA: se aplica un tipo medio del I 5,5\% siguiendo estimaciones obtenidas en consultas con expertos en fiscalidad.

- Otros impuestos: se estiman según cifras oficiales.

- $\quad$ Seguridad Social: Se ha estimado una cotización promedio de 39,3\% del salario bruto anual.

- Aportación de riqueza: se estima en función de la aportación de cada componente a la demanda agregadas del país y su contribución estimada al PIB en forma de consumo privado ( $\mathrm{y}$

5. La demanda agregada de la economía es una forma de relacionar el ingreso y gasto que se produce en un país con los diferentes fines por los que se demanda la producción y en concreto el Producto interior bruto (PIB). La demanda agrega da está formada por la suma de: Consumo privado + Inversión + Gasto público + Saldo exterior (Exportaciones - Importaciones). En este trabajo se ha estimado un efecto indirecto como aportación de riqueza, que se convierte en consumo privado. Dichas estimaciones se han realizado de manera diferente cuando el origen es una persona física (genera consumo de manera indirecta) o jurídica (empresas, proveedores, etc.). Dichas estimaciones se han realizado además teniendo en cuenta la situación económica actual y la información obtenida en el trabajo de campo. sus efectos correlativos en generación de empleo, impuestos, etc.). Se ha estimado un efecto variable entre ( $6,5 \%$ a $33 \%$ ), según el impacto de cada componente.

Las fórmulas básicas de cálculo, con arreglo a las condiciones indicadas, han sido las siguientes:

Inversión Pública $(I P)=C s+S u+I n$, donde $C s=$ Coste de Servicios que asume la Administración. $S u=$ Subvenciones a la persona (por ejemplo, en ayudas técnicas) o a la entidad que le presta servicios. In = Incentivos a la persona o a las empresas, por ejemplo, para la contratación.

Retorno $(R)=I m+S S+A r$, donde Im $=$ Impuestos recaudados. $S S=$ Seguridad Social recaudada. $A r=$ Aportación de riqueza (estimada en términos de aportación estimada al PIB, como parte de la demanda agregada).

Balance $=R-I P$.

$S R O I=(R-I P / I P) * I O O$,

Como expresión de la cantidad

retornada por cada roo euros invertidos.

\section{Resultados}

Tal como se ha indicado en la metodología, se presentan los resultados de retorno de la inversión que realiza el Estado en dos usuarios "tipo" de los perfiles seleccionados. No es por tanto una información generalizable en términos de representatividad estadística, aunque sí resulta significativa dentro del alcance exploratorio del presente estudio.

El punto de partida de este trabajo radica en la definición de un perfil medio del participante en el Programa de Apoyo a la Vida Independiente 
de la Comunidad de Madrid (PAVI), que pueda ser comparado con otro caso similar que cuente con un sistema de apoyos de tipo residencial, dentro de la misma comunidad.

\subsection{Selección de casos de estudio}

La selección de cada uno de los casos de estudio responde a un perfil que viene marcado por las características más prevalentes en cada uno de los servicios que se pretende comparar.

El Programa de Apoyo a la Vida Independiente de la Comunidad de Madrid ofrece asistencia personal a 62 personas con discapacidad física y alto nivel de dependencia (el 90\% de los participantes tiene reconocido el Grado III niveles I y 2 en el Sistema para la Autonomía y Atención a la Dependencia) ${ }^{6}$. De ellas, el $56 \%$ son varones. Uno de los requisitos para el acceso al Programa es contar con una vida activa; así, se observa que el $47 \%$ de los usuarios trabaja, un $37 \%$ estudia, el 10 \% estudia y trabaja y un $6 \%$ se dedica a otras actividades tales como la responsabilidad asociativa. El promedio de edad es de 39 años y en su mayoría cuentan con formación universitaria.

Con estos datos se ha establecido un perfil medio de usuario de la OVI: varón, en torno a 40 años, con discapacidad física y alto nivel de dependencia, con estudios universitarios y que se encuentra trabajando.

Por lo que respecta a la población que recibe apoyos en residencia, se ha seleccionado como caso de estudio el servicio residencial que gestiona la Fundación del Lesionado Medular (FLM) en la Comunidad de Madrid. De acuerdo con los datos proporcionados por el propio centro, para este año (20I3) se contabilizan 60 personas residentes, de las que el $73 \%$ son varones. El promedio de edad es de 48 años y el promedio de estancia de los residentes se sitúa en los 4 años. Respecto a su situación de dependencia, los datos indican que el $67 \%$ de los residentes tiene

6. Datos correspondientes a enero de 2013 , obtenidos de la propia entidad gestora del programa. reconocido el máximo grado de dependencia y que el $93 \%$ no tiene actividad laboral.

Teniendo en cuenta el perfil medio de usuario del servicio residencial, se ha seleccionado un varón de 44 años, residente en la Fundación Lesionado Medular (FLM) desde hace 3 años y en situación laboral inactiva.

\subsection{SROI del perfil de usuario de servicio residencial}

La Tabla 4 recoge las cifras del usuario que recibe apoyos en el centro residencial referidas a las variables que consideramos en la matriz de análisis de impacto en el apartado de la metodología.

Los resultados obtenidos para el usuario del servicio residencial analizado arrojan una inversión pública anual de casi 43.000 euros anuales, exclusivamente para financiar el servicio (que incluye, como se ha indicado, el servicio de rehabilitación). De estos, algo más de 32.000 euros retornan al Estado, a través de impuestos, cotizaciones a la Seguridad Social y otros efectos indirectos. El resultado SROI para el servicio de residencia ha sido de una recuperación de 76,26 euros por cada Ioo que se invierten (ver Tabla 5).

Si se considera la globalidad de ingresos y gastos movilizados en torno al usuario del servicio residencial (es decir, los que tienen que ver con su actividad económica más allá del servicio de asistencia que recibe), la inversión pública anual asciende a casi 53.000 euros anuales, de los que 40.400 retornan al Estado. El resultado SROI para la persona usuaria de servicio de residencia ha sido de una recuperación de casi 76,36 euros de cada roo que se invierten, tal como se resume en la Tabla 6.

\subsection{SROI del perfil de usuario del servicio de asistencia personal}

La Tabla 7 recoge las cifras del usuario que recibe apoyos de asistencia personal a 
Tabla 4. Mapa de impacto económico del usuario tipo de servicios residenciales

\begin{tabular}{|c|c|c|c|c|c|c|c|}
\hline \multirow{3}{*}{ Variables } & \multirow{3}{*}{ Base } & \multicolumn{6}{|c|}{ Impacto Erario público (Monetización) } \\
\hline & & \multirow[b]{2}{*}{ Gasto } & \multicolumn{5}{|c|}{ Retorno } \\
\hline & & & $\begin{array}{l}\text { Impuesto } \\
\text { directo }\end{array}$ & $\begin{array}{c}\text { Otros } \\
\text { impuestos }\end{array}$ & $\begin{array}{l}\text { Seguridad } \\
\text { Social }\end{array}$ & $\begin{array}{l}\text { Aportación } \\
\text { de riqueza }\end{array}$ & $\begin{array}{c}\text { Otros } \\
\text { retornos }\end{array}$ \\
\hline Ingresos & $10.080,00$ & $7.080,00$ & $2.289,90$ & 0,00 & 0,00 & 0,00 & 0,00 \\
\hline Empleo & 0,00 & 0,00 & 0,00 & 0,00 & 0,00 & 0,00 & 0,00 \\
\hline Prestaciones Económicas: & $7.080,00$ & $7.080,00$ & $1.539,90$ & 0,00 & 0,00 & 0,00 & 0,00 \\
\hline Otros ingresos: & $3.000,00$ & 0,00 & 750,00 & 0,00 & 0,00 & 0,00 & 0,00 \\
\hline Gastos & $13.195,60$ & $2.972,20$ & $1.141,01$ & $2.108,91$ & 0,00 & $2.177,27$ & 0,00 \\
\hline $\begin{array}{l}\text { Gastos especiales por } \\
\text { discapacidad }\end{array}$ & $5.246,00$ & $2.972,20$ & $1.141,01$ & 813,13 & 0,00 & 865,59 & 0,00 \\
\hline Consumo & $7.949,60$ & 0,00 & 0,00 & $1.295,78$ & 0,00 & $1.311,68$ & 0,00 \\
\hline $\begin{array}{l}\text { Servicios de Apoyo en la } \\
\text { vida diaria }\end{array}$ & $42.858,30$ & $\left(^{* \star * *}\right) 42.858,30$ & $\left.{ }^{* \star}\right) 5.537,08$ & $(* *) 2.702,86$ & $\left.{ }^{* \star}\right) 13.171,47$ & $(* *) 11.272,16$ & 0,00 \\
\hline Amortización ${ }^{1}$ & $1.285,75$ & $1.285,75$ & 0,00 & 205,08 & 0,00 & 212,15 & 0,00 \\
\hline RRHH (atención directa) & $22.714,90$ & $22.714,90$ & $4.940,49$ & 0,00 & $8.926,96$ & $7.495,92$ & 0,00 \\
\hline RRHH ( gestión) & $2.742,93$ & $2.742,93$ & 596,59 & 0,00 & $1.077,97$ & 905,17 & 0,00 \\
\hline Alquileres & 0,00 & 0,00 & 0,00 & 0,00 & 0,00 & 0,00 & 0,00 \\
\hline Suministros, & $12.707,49$ & $12.707,49$ & 0,00 & $1.969,66$ & $2.497,02$ & $2.096,74$ & 0,00 \\
\hline Mantenimiento & $3.407,23$ & $3.407,23$ & 0,00 & 528,12 & 669,52 & 562,19 & 0,00 \\
\hline Otros (seguros, etc.) & 0,00 & 0,00 & 0,00 & 0,00 & 0,00 & 0,00 & 0,00 \\
\hline TOTAL & & $\left(^{* \star *}\right) 52.910,50$ & (*) $8.967,98$ & (*) $4.811,77$ & $\left(^{\star}\right) 13.171,47$ & (*) $13.449,44$ & 0,00 \\
\hline
\end{tabular}

${ }^{1}$ Amortización anual = coste de la obra / 33 (años promedio en que se considera amortizada la infraestructura según lo establecido en el Real Decreto $1777 / 2004$, de 30 de julio, por el que se aprueba el Reglamento del Impuesto sobre Sociedades).

(****) Inversión pública en asistencia $(42.858,30$ euros/año).

(***) Inversión pública total (52.910,50 euros/año).

(**) Retorno en servicios de asistencia (32.683,57 euros/año).

(*) Retorno total (40.400,66 euros/año).

Fuente: Elaboración propia, a partir de criterios de estimación explicados en metodología.

Tabla 5. Resultado SROI en servicio residencial, por persona tipo y año

\begin{tabular}{|l|r|l|}
\hline INVERSIÓN PÚBLICA & $\mathbf{4 2 . 8 5 8 , 3 0}$ & Euros \\
\hline RETORNO & $\mathbf{3 2 . 6 8 3 , 5 7}$ & Euros \\
\hline BALANCE & $-\mathbf{1 0 . 1 7 4 , 7 3}$ & Euros \\
\hline SROI x 100 & $-\mathbf{2 3 , 7 4}$ & Porcentaje \\
\hline $\begin{array}{l}\text { Por cada 100 } \\
\text { invertidos }\end{array}$ & $\mathbf{7 6 , 2 6}$ & $\begin{array}{l}\text { Euros de } \\
\text { retorno }\end{array}$ \\
\hline
\end{tabular}

Fuente: Elaboración propia, a partir del trabajo de campo realizado.
Tabla 6. Resultado total SROI en servicio residencial por persona tipo y año (incluye flujo monetario del usuario, además del servicio)

\begin{tabular}{|l|r|l|}
\hline INVERSIÓN PÚBLICA & $\mathbf{5 2 . 9 1 0 , 5 0}$ & Euros \\
\hline RETORNO & $\mathbf{4 0 . 4 0 0 , 6 6}$ & Euros \\
\hline BALANCE & $\mathbf{- 1 2 . 5 0 9 , 8 4}$ & Euros \\
\hline SROI x 100 & $-\mathbf{2 3 , 6 4}$ & Porcentaje \\
\hline $\begin{array}{l}\text { Por cada 100 } \\
\text { invertidos }\end{array}$ & $\mathbf{7 6 , 3 6}$ & $\begin{array}{l}\text { Euros de } \\
\text { retorno }\end{array}$ \\
\hline
\end{tabular}

Fuente: Elaboración propia, a partir del trabajo de campo realizado. 
Tabla 7. Mapa de impacto económico del usuario tipo del Programa de Apoyo a la Vida Independiente (PAVI)

\begin{tabular}{|c|c|c|c|c|c|c|c|}
\hline \multirow{3}{*}{ Variables } & \multirow{3}{*}{ Base } & \multicolumn{6}{|c|}{ Impacto Erario público (Monetización) } \\
\hline & & \multirow[b]{2}{*}{ Gasto } & \multicolumn{5}{|c|}{ Retorno } \\
\hline & & & $\begin{array}{l}\text { Impuesto } \\
\text { directo }\end{array}$ & $\begin{array}{c}\text { Otros } \\
\text { impuestos }\end{array}$ & $\begin{array}{l}\text { Seguridad } \\
\text { Social }\end{array}$ & $\begin{array}{c}\text { Aportación de } \\
\text { riqueza }\end{array}$ & $\begin{array}{l}\text { Otros } \\
\text { retornos }\end{array}$ \\
\hline Ingresos & $25.200,00$ & $9.576,00$ & $5.481,00$ & 0,00 & $9.903,60$ & 0,00 & 0,00 \\
\hline Empleo & $25.200,00$ & $9.576,00$ & $5.481,00$ & 0,00 & $9.903,60$ & 0,00 & 0,00 \\
\hline Prestaciones Económicas: & 0,00 & 0,00 & 0,00 & 0,00 & 0,00 & 0,00 & 0,00 \\
\hline Otros ingresos: & 0,00 & 0,00 & 0,00 & 0,00 & 0,00 & 0,00 & 0,00 \\
\hline Gastos & $23.793,00$ & $2.375,10$ & $2.042,98$ & $3.803,12$ & 0,00 & $3.925,85$ & 0,00 \\
\hline $\begin{array}{l}\text { Gastos especiales por } \\
\text { discapacidad }\end{array}$ & $9.393,00$ & $2.375,10$ & $2.042,98$ & $1.455,92$ & 0,00 & $1.549,85$ & 0,00 \\
\hline Consumo & $14.400,00$ & 0,00 & 0,00 & $2.347,20$ & 0,00 & $2.376,00$ & 0,00 \\
\hline $\begin{array}{l}\text { Servicios de Apoyo en la } \\
\text { vida diaria }\end{array}$ & $23.099,76$ & $\left(^{\star \star \star \star}\right) 23.099,76$ & $\left.{ }^{(* \star}\right) 4.848,35$ & $\left.{ }^{* \star}\right) 60,87$ & $\left.{ }^{(* *}\right) 8.837,63$ & ${ }^{(* *)} 7.420,91$ & $(* \star) 0,00$ \\
\hline Amortización & 0,00 & 0,00 & 0,00 & 0,00 & 0,00 & 0,00 & 0,00 \\
\hline RRHH (atención directa) & $21.251,78$ & $21.251,78$ & $4.622,26$ & 0,00 & $8.351,95$ & $7.013,09$ & 0,00 \\
\hline RRHH (gestión) & $1.039,49$ & $1.039,49$ & 226,09 & 0,00 & 408,52 & 343,03 & 0,00 \\
\hline Alquileres & 0,00 & 0,00 & 0,00 & 0,00 & 0,00 & 0,00 & 0,00 \\
\hline Suministros, & 392,70 & 392,70 & 0,00 & 60,87 & 77,16 & 64,79 & 0,00 \\
\hline Mantenimiento & 0,00 & 0,00 & 0,00 & 0,00 & 0,00 & 0,00 & 0,00 \\
\hline Otros (seguros, etc.) & 415,80 & 415,80 & 0,00 & 0,00 & 0,00 & 0,00 & 0,00 \\
\hline \multicolumn{2}{|l|}{ TOTAL } & $(* \star *) 35.050,86$ & (*) $12.372,33$ & (*) $3.863,98$ & (*) $18.741,23$ & (*) $11.346,76$ & $(*) 0,00$ \\
\hline \multicolumn{8}{|c|}{$\begin{array}{l}(* * * *) \text { Inversión pública en asistencia (23.099,76 euros/año). } \\
(* * *) \text { Inversión pública total (35.050,86 euros/año). } \\
(* *) \text { Retorno en servicios de asistencia (2I.I67,77 euros/año). } \\
(*) \text { Retorno total ( } 46.324,3 \text { I euros/año). } \\
\text { Funten: Elaboración propia, a partir de criterios de estimación }\end{array}$} \\
\hline
\end{tabular}

través del PAVI, referidas a las variables que consideramos en la matriz de análisis de impacto en el apartado de la metodología "Variables y fórmulas para monetización".

Los resultados obtenidos para el usuario del PAVI, arrojan una inversión pública anual de 23. I00 euros anuales, exclusivamente para financiar el servicio de asistencia personal. De éstos, algo más de 2 I.000 retornan al Estado. El resultado SROI para el PAVI ha sido de una recuperación de casi 92 euros de cada roo que se invierten (Tabla 8).

Si se considera la globalidad de ingresos y gastos movilizados en torno al usuario del servicio de asistencia personal (es decir, los que tienen que
Tabla 8. Resultado SROI en servicios del PAVI por usuario tipo y año

\begin{tabular}{|l|r|l|}
\hline INVERSIÓN PÚBLICA & $\mathbf{2 3 . 0 9 9 , 7 6}$ & Euros \\
\hline RETORNO & $\mathbf{2 1 . 1 6 7 , 7 7}$ & Euros \\
\hline BALANCE & $-1.931,99$ & Euros \\
\hline SROI x 100 & $-8,36$ & Porcentaje \\
\hline $\begin{array}{l}\text { Por cada } \\
100 \text { invertidos }\end{array}$ & $\mathbf{9 1 , 6 4}$ & $\begin{array}{l}\text { Euros de } \\
\text { retorno }\end{array}$ \\
\hline
\end{tabular}

Fuente: Elaboración propia, a partir del trabajo de campo realizado. 
ver con su actividad económica más allá del servicio), la inversión pública anual asciende a 35.000 euros anuales, que generan un retorno de más de 46.000 al Estado. El resultado SROI para la persona usuaria de servicio de asistencia personal ha sido la única de las situaciones analizadas que arroja un saldo positivo de I32 euros de retorno por cada Ioo euros que se invierten (Tabla 9).

Tal como se refleja en los gráficos siguientes, es preciso considerar los dos servicios analizados en el contexto personal de cada usuario, para adquirir una perspectiva global sobre los resultados de la Inversión Pública.
Tabla 9. Resultado total SROI en servicios del PAVI por usuario tipo y año (incluye flujo monetario del usuario, además del servicio)

\begin{tabular}{|l|r|l|}
\hline INVERSIÓN PÚBLICA & $\mathbf{3 5 . 0 5 0 , 8 6}$ & Euros \\
\hline RETORNO & $\mathbf{4 6 . 3 2 4 , 3 1}$ & Euros \\
\hline BALANCE & $\mathbf{1 1 . 2 7 3 , 4 4}$ & Euros \\
\hline SROI x 100 & $\mathbf{3 2 , 1 6}$ & Porcentaje \\
\hline $\begin{array}{l}\text { Por cada 100 } \\
\text { invertidos }\end{array}$ & $\mathbf{1 3 2 , 1 6}$ & $\begin{array}{l}\text { Euros de } \\
\text { retorno }\end{array}$ \\
\hline
\end{tabular}

Fuente: Elaboración propia, a partir del trabajo de campo realizado.

Gráfico 1. Inversión pública y retorno al Estado de los servicios de apoyo (de asistencia personal y de residencia). Euros por usuario tipo al año

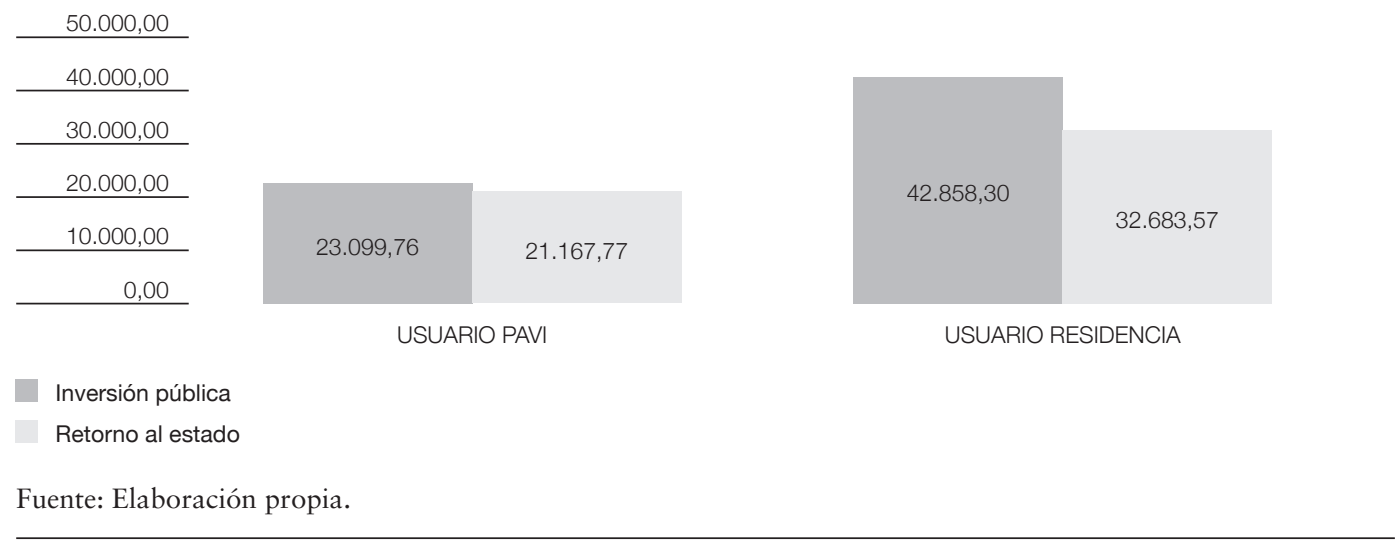

Gráfico 2. Inversión pública y retorno al Estado por cada usuario tipo (de asistencia personal y de residencia), considerando totalidad de ingresos y gastos. Total euros por usuario tipo al año

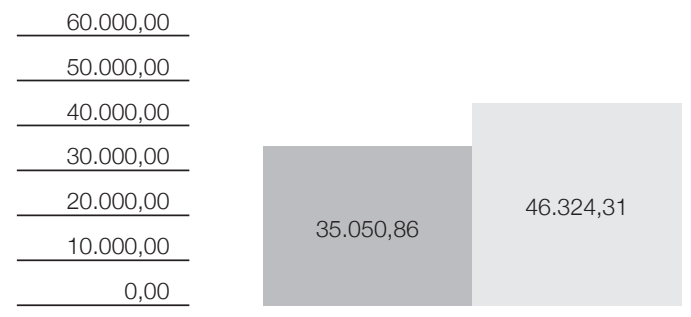

USUARIO PAVI TOTAL

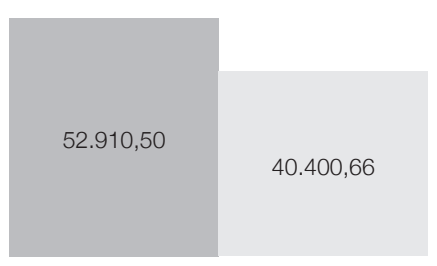

USUARIO RESIDENCIA TOTAL

Inversión pública

Retorno al estado

Fuente: Elaboración propia. 
Gráfico 3. Resultado de la inversión en retorno para el Estado. Euros al año (por cada 100 invertidos)

$\begin{array}{r}140,00 \\ \hline 120,00 \\ \hline 100,00 \\ \hline 80,00 \\ \hline 60,00 \\ \hline 40,00 \\ \hline 20,00 \\ \hline 0,00 \\ \hline\end{array}$

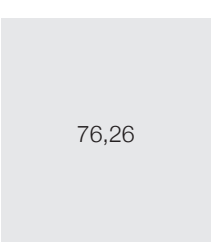

USUARIO
RESIDENCIA

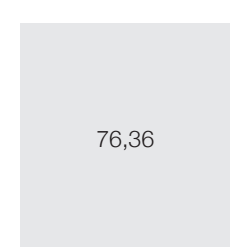

USUARIO RESIDENCIA TOTAL

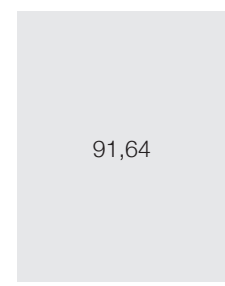

USUARIO PAVI

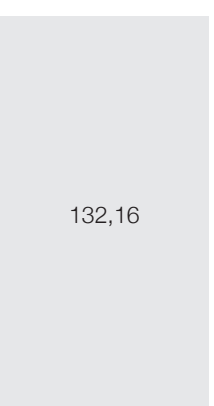

USUARIO PAVI

Fuente: Elaboración propia.

Gráfico 4. Índice SROI en términos porcentuales respecto a la inversión realizada por el Estado (SROI x 100)

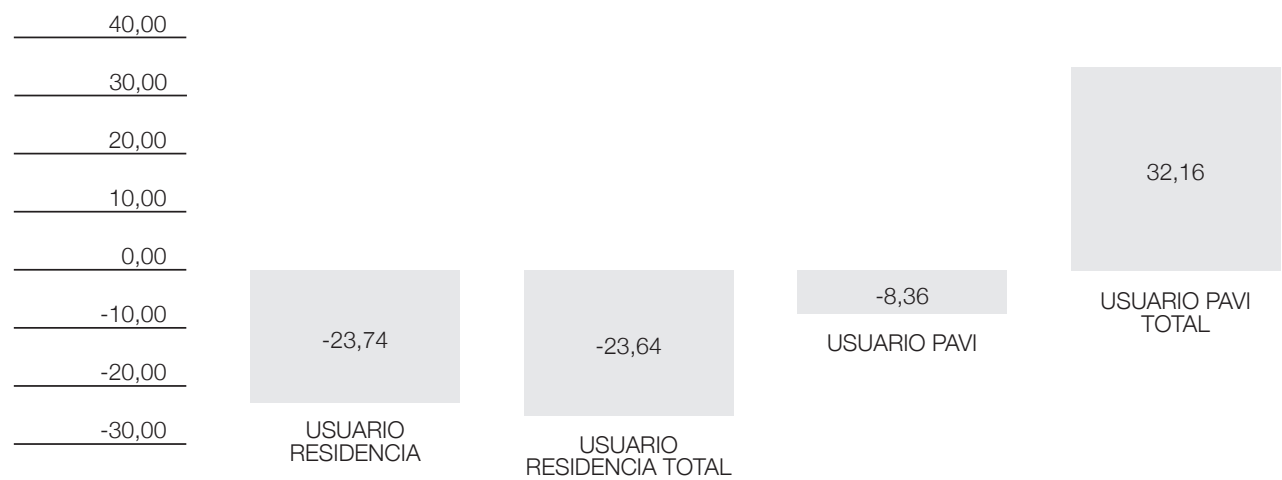

Fuente: Elaboración propia.

En general, el uso de servicios de la comunidad, unida a un despliegue menor de recursos (públicos y privados) en términos absolutos, sitúa a los servicios de asistencia personal como una opción socialmente más rentable, siempre en combinación con una situación laboralmente activa.
3.4. Valores y desafíos de la asistencia personal y la atención residencial en la voz de los protagonistas

Existen aspectos que no se pueden expresar en términos monetarios y que sin embargo crean valor social. Los cuadros I y 2 recogen con brevedad expresiones que refieren estos valores y desafíos relacionados con la autoestima, el decidir sobre la propia vida, el contar con un entorno de seguridad, el crear establecer redes familiares y de apoyo, difíciles de convertir a términos económicos. 


\begin{tabular}{|c|c|}
\hline Aspectos positivos & Desafíos \\
\hline 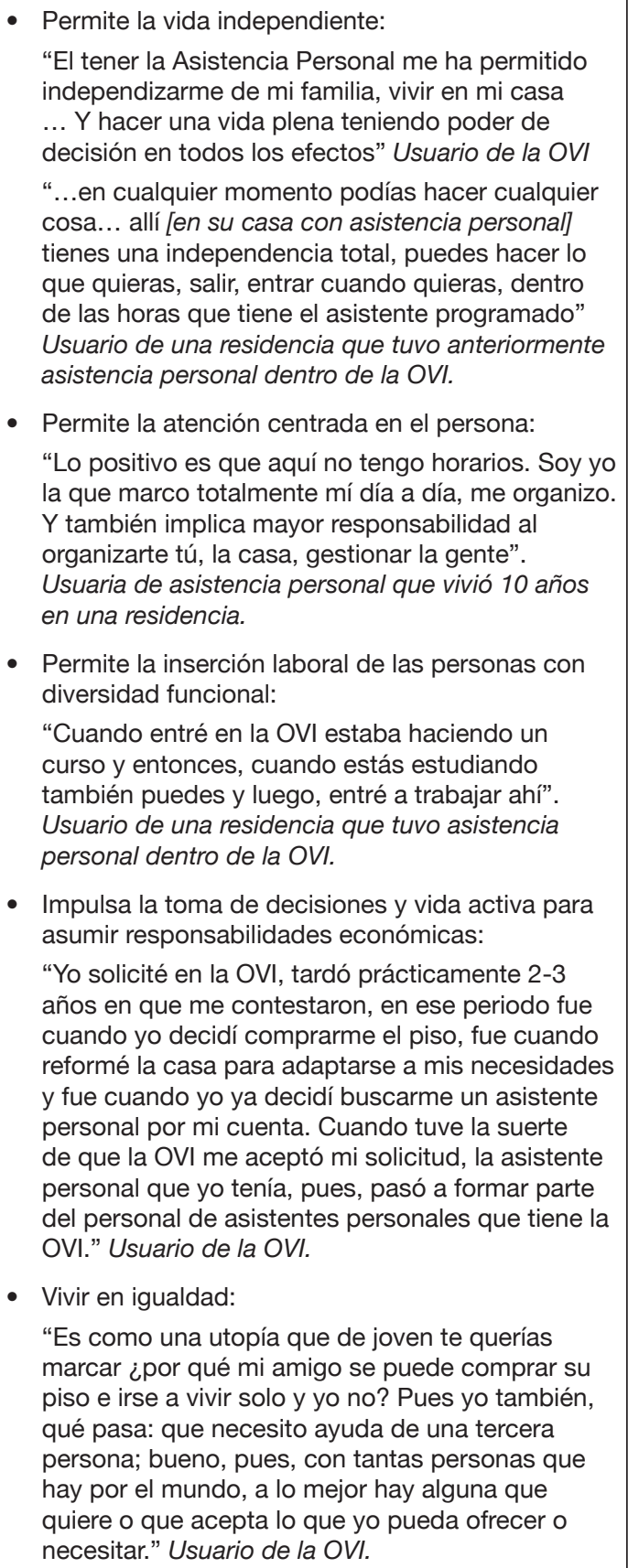 & $\begin{array}{l}\text { - Fortalecer las redes de apoyo: } \\
\text { "El primer momento en casa, pues sí, mis } \\
\text { hermanos me sirvieron de apoyo. Pero llega un } \\
\text { momento que ellos también hacen su vida y te va } \\
\text { faltando gente alrededor". Usuario de residencia. } \\
\text { - La accesibilidad del entorno incluyendo la } \\
\text { vivienda, el barrio, la ciudad, los transportes: } \\
\text { "Para que alguna persona tenga asistente } \\
\text { personal, evidentemente hay otro pilar importante } \\
\text { que es tener una casa, un medio donde poder } \\
\text { estar. Porque por mucho asistente que tengas, } \\
\text { si no tienes un sitio para vivir... Yo no puede vivir } \\
\text { en una casa en que el baño no está adaptado, o } \\
\text { que haya escaleras para entrar al portal" Usuaria } \\
\text { de asistencia personal que vivió } 10 \text { años en una } \\
\text { residencia. } \\
\text { "Hasta cierto punto la inclusión no es solo tener la } \\
\text { casa y el recurso, sino también es tener una zona } \\
\text { que tenga servicios, que la gente pueda bajar a } \\
\text { la calle y tener un medio de vida como cualquier } \\
\text { otro, porque el desplazamiento para nosotros es } \\
\text { mucho más difícil". Usuaria de asistencia personal } \\
\text { que vivió } 10 \text { años en una residencia. } \\
\text { - Impulsar el empoderamiento de las personas } \\
\text { con discapacidad, (fomentar la filosofía de vida } \\
\text { independiente...), contar con un entorno (familiar) } \\
\text { que promueva la responsabilidad y la toma de } \\
\text { decisiones: } \\
\text { "Yo estoy aquí también, gracias a ellos [los } \\
\text { padres]. Ellos me han permitido la mentalidad que } \\
\text { tengo para luchar por ser independiente, entre } \\
\text { comillas, por el tema de depender de segundas } \\
\text { personas, pero sí por lo menos de decidir lo que } \\
\text { yo quiero, me equivoque o no me equivoque." } \\
\text { Usuario de la OVI. }\end{array}$ \\
\hline
\end{tabular}

Fuente: Elaboración propia, a partir del trabajo de campo realizado. 
Cuadro 2. Aspectos positivos y desafíos del servicio residencial desde las voces de los protagonistas

\begin{tabular}{|c|c|}
\hline Aspectos positivos & Desafíos \\
\hline $\begin{array}{l}\text { - Puede ser un recurso de transición hacia la vida } \\
\text { independiente: } \\
\text { "El motivo mío de la residencia era la inclusión } \\
\text { porque estaba estudiando, empezando a trabajar, } \\
\text { tenía una vida totalmente activa. No era una persona } \\
\text { que para mí la residencia fuera un lugar para vivir, } \\
\text { era un medio para conseguir otra vida". Usuaria } \\
\text { de asistencia personal que vivió } 10 \text { años en una } \\
\text { residencia. } \\
\text { - Puede ser un servicio de apoyo: } \\
\text { "Yo hice el Máster gracias a la residencia porque } \\
\text { si no, no hubiera podido". Usuaria de asistencia } \\
\text { personal que vivió } 10 \text { años en una residencia. } \\
\text { - Acoge a aquellas personas que han perdido sus } \\
\text { redes de apoyo: } \\
\text { "Mi hermana tuvo que dejar el piso y entonces, yo } \\
\text { me quedé en la calle". Usuario de una residencia que } \\
\text { tuvo asistencia personal dentro de la OVI. } \\
\text { "El principal motivo de que yo viniera aquí fue que } \\
\text { falleció mi madre de repente" Usuario de residencia. } \\
\text { - Permite que las personas con discapacidad vivan en } \\
\text { un entorno accesible: } \\
\text { "Con mi familia está complicado porque montar en } \\
\text { los coches de ellos pues tampoco puedo. Y luego, } \\
\text { las casas solo hay una que es accesible; vamos, } \\
\text { accesible al salón, entrar al salón, tienes que ir de } \\
\text { visita y volver" Usuario de residencia. }\end{array}$ & $\begin{array}{l}\text { - Rigidez: } \\
\text { "La restricción. Ahí tienes como pautado } \\
\text { el número de duchas a la semana, tanto te } \\
\text { vengan bien como no... Luego, los días que si } \\
\text { llevas el pelo largo, cosa que yo siempre llevo } \\
\text { y llevaré, había como indirectas: "¿Cómo no } \\
\text { te lo cortas?"; siempre la comodidad. Bueno, } \\
\text { me lo cortaré si a mí me apetece". Usuaria de } \\
\text { asistencia personal que vivió } 10 \text { años en una } \\
\text { residencia. } \\
\text { - Preservar la intimidad (mujeres): } \\
\text { "Hay veces que allí el derecho de intimidad no } \\
\text { está bien regulado, y eso es importantísimo. Por } \\
\text { ejemplo, para una chica joven, al principio, que } \\
\text { llegara un chico como un cuidador pues era } \\
\text { muy violento." Usuaria de asistencia personal } \\
\text { que vivió } 10 \text { años en una residencia. } \\
\text { - Promocionar la vida activa: } \\
\text { "las residencias, para según qué personas, no } \\
\text { les ayudan a tomar las riendas de su propia } \\
\text { vida porque como te organizan todo..." Usuaria } \\
\text { de asistencia personal que vivió } 10 \text { años en una } \\
\text { residencia. } \\
\text { - Evitar la sobreprotección: } \\
\text { "La mayoría hacen talleres porque yo creo que } \\
\text { fuera no se encuentran tan protegidos". Auxiliar } \\
\text { de residencia. } \\
\text { Cierto aislamiento: } \\
\text { "No sales tanto porque para salir tienes que } \\
\text { salir tú solo, tienes que tener a alguien que te } \\
\text { acompañe en la mayoría de los casos." Usuario } \\
\text { de una residencia que tuvo asistencia personal } \\
\text { dentro de la OVI. }\end{array}$ \\
\hline
\end{tabular}

Fuente: Elaboración propia, a partir del trabajo de campo realizado.

\section{Discusión}

Este trabajo plantea una aproximación al impacto económico directo e indirecto en términos monetarios de dos casos tipo de una persona con discapacidad que recibe servicio de asistencia personal, respecto a otra que recibe servicio de apoyo en un centro residencial. A partir de la metodología aplicada y sus resultados, cabe apuntar las siguientes consideraciones:
- $\quad$ Según se constató como parte de la investigación del marco contextual, a pesar del impulso que supone la Convención de los Derechos de las Personas con Discapacidad (CDPD), como instrumento jurídico vinculante, y los avances correspondientes en el ámbito europeo de políticas, así como de tratarse de una figura recogida en la Ley 39/2006, de I 4 de diciembre, de Promoción de la Autonomía Personal y Atención a las personas en situación de dependencia, la Asistencia 
Personal cuenta con una implantación todavía incipiente en nuestro país. Aunque existen iniciativas muy relevantes como el Programa de Apoyo a la Vida Independiente (PAVI) de la Comunidad de Madrid, su participación es sumamente reducida (en torno al o, I por ciento sobre el total de las prestaciones del SAAD) en términos cuantitativos respecto a otras modalidades de asistencia.

- En cuanto a los resultados de Retorno Social de la Inversión (SROI) para el caso del servicio residencial estudiado, sobre la inversión pública anual realizada exclusivamente para financiar el servicio (cerca de 43.000 euros) se estima un retorno al Estado a través de impuestos, Seguridad Social y aportación de riqueza vía consumo de 76,26 euros por cada Ioo invertidos. Si se considera la globalidad de ingresos y gastos movilizados en torno a este usuario (es decir, los que tienen que ver con su actividad económica más allá del servicio), el retorno al Estado de la inversión pública anual se mantiene en torno a los 76 euros por cada roo que se invierten.

- A partir de los resultados obtenidos para el usuario de asistencia personal, sobre la inversión pública anual realizada en exclusiva para financiar el servicio (23.100 euros), se estima una recuperación o retorno al Estado de casi 92 euros de cada roo de inversión. Al considerar el conjunto de ingresos y gastos movilizados en torno al usuario del servicio de asistencia personal, el resultado de la inversión pública arroja un saldo positivo de $\mathrm{I} 32$ euros de retorno por cada roo euros invertidos.

- Aun constatando una notable tasa de retorno de la inversión pública en ambos servicios, ésta resulta más elevada en el caso de la asistencia personal, que también supone un mayor retorno social a menor despliegue de inversión pública en términos absolutos. La tasa de retorno es aún mayor al considerar el contexto personal de cada usuario, su actividad laboral (cuando se da) y en general el uso de bienes y servicios de la comunidad.

- Por otro lado, se comprueba que dentro de las condiciones actuales para el acceso a la asistencia personal o a la atención en residencia interviene una serie de factores sociales, como el contar o no con una red de apoyos informales, el acceso a una vivienda, un nivel de estudios (en curso o realizados) entre medio y alto, que abra opciones al desempeño laboral, (formación en) competencias para gestionar la asistencia personal, además de otros condicionantes de tipo personal.

- Más allá de los resultados de retorno social que se han identificado y convertido a términos económicos, existen otros aspectos y valores que puede promover un servicio, fundamentales para usuarios, gestores y decisores políticos, como la autoestima, la opción de decidir sobre la propia vida, el establecer o mantener relaciones familiares, la calidad de vida percibida, que no son expresables en términos monetarios. Se han recogido expresiones indicativas en este sentido, en la voz de los protagonistas.

- Es importante tener en cuenta el carácter aproximativo y exploratorio del presente trabajo, basado en un estudio de caso a partir de un perfil «tipo» de persona usuaria de asistencia personal a través del PAVI y un perfil análogo en el ámbito de la atención residencial. Los resultados que se presentan, susceptibles de ampliar a futuro mediante otras investigaciones sobre impacto de la inversión son indicativos, aunque no responden a criterios de representatividad estadística.

- Conviene tener en cuenta además que dentro de las condiciones que influyen en las personas con discapacidad para el acceso a los servicios, intervienen una serie de factores sociales, como el disponer de una red de apoyos informales, acceso a una vivienda accesible, nivel de estudios, 
desempeño laboral y otros condicionantes de tipo personal (Hurstfield, Parasha y Schofield, 2007).

- $\quad$ El presente estudio no pretende favorecer un servicio frente a otro. El equipo de investigación considera fundamental, en todo caso, promover la inclusión comunitaria y afianzar la posibilidad de opción entre estos servicios, en concordancia con la CDPD, tal como lo expresaba una de las personas con discapacidad entrevistada: "lo importante es tener el derecho a elegir lo que tú quieras".

Referencias bibliográficas

Arnau, S. et al. (2007): “Asistencia Personal para la Vida Independiente y la promoción de la autonomía de las personas con diversidad funcional (discapacidad) en España”. (en línea). <http://www.faeba.es/descargar.php?id=5>, acceso 27 de noviembre de $20 \mathrm{I} 2$.

Comisión Europea (2010): "Estrategia Europea sobre Discapacidad 2010-2020: un compromiso renovado para una Europa sin barreras". Comunicación de la Comisión al Parlamento Europeo, al Consejo, al Comité Económico y Social Europeo y al Comité de las Regiones. COM (2010) 636 final. Bruselas, I 5.I I.20Io. (en línea). <http://goo.gl/ $\mathrm{WgEFB}_{4}>$, acceso 20 de noviembre de $20 \mathrm{I} 2$.

Consejería de Asuntos Sociales (20I 2): III Plan de Acción para personas con discapacidad 2012 20I5. Madrid: Comunidad de Madrid. (en línea). <http://goo.gl/ixXOv5 >, acceso 27 de noviembre de $20 \mathrm{I} 2$.

ECODES. Alter Civites (20I2): Análisis del retorno económico directo de la inversión pública en CEE de iniciativa social mediante aplicación de la metodología SROI (Retorno Social de las Inversiones). Grupo Gureak, Tasubinsa y la Asociación de Centros Especiales de Empleo de Navarra (ACEMNA). (en línea). <http://www. acemna.es/INFORME_RetornoCEE_Fase I. pdf $>$, acceso I I de noviembre de 20 I 2.
España. Real Decreto I97I/I999, de 23 de diciembre, de procedimiento para el reconocimiento, declaración y calificación del grado de minusvalía. Boletín Oficial del Estado, núm. 22 de 26 de enero de 2000 , p. 3317-34IO.

España. Real Decreto Legislativo 3/2004, de 5 de marzo, por el que se aprueba el texto refundido de la Ley del Impuesto sobre la Renta de las Personas Físicas. Boletín Oficial del Estado, núm. 60 de ro de marzo de 2004, p. 10670-1072I.

España. Real Decreto I777/2004, de 30 de julio, por el que se aprueba el Reglamento del Impuesto sobre Sociedades. Boletín Oficial del Estado, núm. I 89, de 6 de agosto de 2004, p. $28377-28429$.

España. Ley 39/2006, de I4 de diciembre, de la Autonomía Personal y Atención a las Personas en situación de Dependencia. Boletín Oficial del Estado, núm. 299 de I 5 de diciembre de 2006, p. 44I42-44I 56.

España. Real Decreto I74/20I I, de I I de febrero, por el que se aprueba el Baremo de valoración de la situación de dependencia establecido por la Ley 39/2006, de I 4 de diciembre, de Promoción de la Autonomía Personal y Atención a las personas en situación de 
dependencia. Boletín Oficial del Estado, núm. 42 de I 8 de febrero de 20 II, p. I8567-I869I.

Hurstfield J. Parashar U. Schofield K. (2007): The costs and benefits of independent living. London: Office for Disability Issues (ODI). (en línea). <http://xurl.es/4urg4>, acceso Io de octubre de $20 \mathrm{I} 2$.

IMSERSO (20I3): Estadísticas mensuales del SAAD. (en línea). <http://dependencia. imserso.es/dependencia_oI/estadisticas/datos_ estadisticos_saad/index.htm>, acceso el I 3 de abril de 2013 .

Pena Trapero. B (2009): "La medición del Bienestar Social: una revisión crítica" en Estudios de
Economía Aplicada. Vol. 27-2, págs. 299-324.

Rodríguez Cabrero, G. (2009): El Sistema de Autonomía Personal y Atención a la Dependencia en España (SAAD): desarrollo y ámbitos de evaluación. Revista Economistas, Año $\mathrm{N}^{\circ} 27, \mathrm{~N}^{\circ}$ I 22, 2009, págs. 8-I6.

The SROI Network (2OI2): Guía para el Retorno Social de la Inversión (SROI). The Cabinet Office. Reino Unido. Traducido y adaptado al español por GRUPO CIVIS. (en línea). <http:// www.thesroinetwork.org/publications/doc_ details/445-the-sroi-guide-spanish >, acceso I I de noviembre de 2012 . 